\title{
O Comprimento da Trajetória de um Projétil
}

\author{
The projectile path length \\ Antonia D. S. Bruno \\ Departamento de Engenharia Mecânica e Produção, \\ Universidade Federal do Ceará, \\ Caixa Postal 12144, 60455-760, Fortaleza, CE, Brasil \\ J. Mauricio O. Matos \\ Departamento de Física, Universidade Federal do Ceará \\ Caixa Postal 6030, 60451-970, Fortaleza, CE, Brasil \\ mauricio@física.ufc.br
}

Recebido em 16 de outubro, 2001. Aceito em 4 de março, 2002.

\begin{abstract}
Neste trabalho apresentamos o cálculo analítico do comprimento da trajetória de um projétil onde, nas equações do movimento, é incluída uma força de atrito diretamente proporcional à velocidade do projétil. Apesar da presença da força de resistência do ar, é possível resolver analiticamente as equações do movimento e determinar a equação da trajetória e o seu comprimento. Comparamos os resultados obtidos com o modelo parabólico e determinamos o ângulo de lançamento que proporciona o maior comprimento da trajetória.
\end{abstract}

In this work we present the calculation of the path length of a projectile where, in the motion equation, a drag force directly proportional to the velocity of the projectile has been included. Despite of the drag force it is possible to solve the motion equations and determine the path and its length exactly. The obtained results are compared with the parabolic model and the launch angle that gives the greatest path length is computed.

\section{Introdução}

A abordagem clássica do movimento de um projétil lançado da superfície da terra geralmente resolve as equações do movimento e, subseqüentemente, obtém a equação da trajetória, o tempo de vôo, a altura máxima atingida e o alcance. O exemplo mais simples de um estudo desta natureza, apresentado nos cursos introdutórios de física para alunos de física e engenharias, é o do movimento de um projétil que obedece o modelo parabólico de Galileu [1], onde se despreza qualquer influência da atmosfera. Recentemente essa abordagem foi expandida incluindo-se o cálculo do comprimento da trajetória do projétil [2]. Foi mostrado que é possível, a partir da equação da trajetória e mediante uma integração, obter-se uma expressão analítica para o comprimento da trajetória.

Quando se incluem os efeitos da resistência do ar sobre o movimento de um projétil, a solução das equações do movimento torna-se mais difícil e não pode ser obtida analiticamente [3]. No entanto, para lançamentos com baixas velocidades, para valores do número de Reynolds $R_{e}<1.0$, a força de resistência do ar pode ser assumida como diretamente proporcional à velocidade do projétil $[4,5]$ e podemos resolver as equações do movimento analiticamente.

Neste trabalho apresentamos o cálculo analítico do comprimento da trajetória de um projétil assumindo, além da força gravitacional, uma força de resistência diretamente proporcional à velocidade do projétil. Na seção II, apresentamos detalhes do cálculo; na seção III, comparamos os resultados obtidos com os resultados obtidos com o modelo parabólico, e na seção IV apresentamos as conclusões.

\section{Detalhes do cálculo}

Nesta seção mostraremos o cálculo do comprimento da trajetória de um projétil nos modelos parabólico e com resistência do ar.

\section{a) Modelo parabólico}

Na Fig. 1 vemos a relação geométrica entre os ele- 
mentos diferenciais $d S, d x$ e $d y$,

$$
d S=\sqrt{d x^{2}+d y^{2}} .
$$

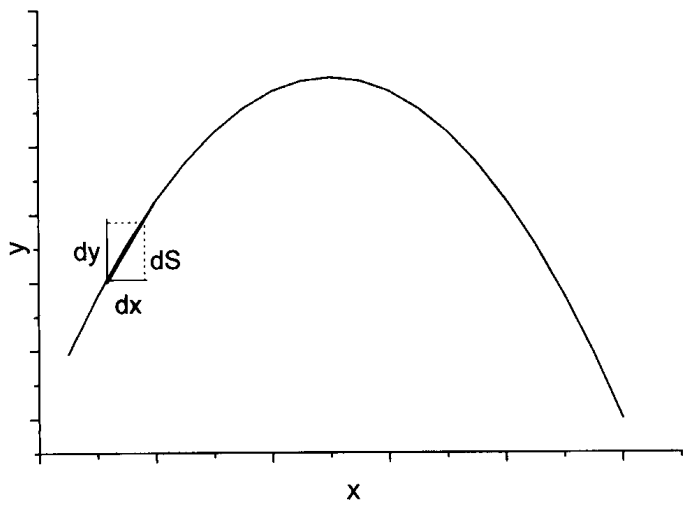

Figura 1. Ilustração da relação geométrica entre $d S, d x e$ $d y$.

A integração de $d S$ segue diretamente

$$
S=\int_{0}^{R} \sqrt{1+\left(\frac{d y}{d x}\right)^{2}} d x,
$$

onde $R$ é o alcance. Conhecendo-se a equação da trajetória do movimento, $S$ é obtida diretamente através da Eq. (01). Observando que $d y / d x=v_{y} / v_{x}$, podemos, alternativamente, determinar $S$ integrando a Eq. (01) no tempo, ou seja: para o modelo parabólico $v_{x}=v_{0} \cos \theta$ e $v_{y}=v_{0} \operatorname{sen} \theta-g t$ onde $v_{0}$ e $\theta$ são respectivamente a velocidade e o ângulo de lançamento. Escrevemos então

$$
\sqrt{1+\left(\frac{d y}{d x}\right)^{2}}=\sqrt{\frac{v^{2}+(u-g t)^{2}}{v^{2}}},
$$

onde $v=v_{0} \cos \theta$ e $u=v_{0} \operatorname{sen} \theta$. A integral definida na Eq. (1) pode ser escrita como

$$
S=\int_{0}^{T} \sqrt{v^{2}+(u-g t)^{2}} d t,
$$

onde $T=2 u / g$ é o tempo de vôo. Fazendo a mudança de variável, $\chi=u-g t$, podemos escrever a Eq. (2) como

$$
S=\frac{2}{g} \int_{0}^{u} \sqrt{v^{2}+\chi^{2}} d \chi .
$$

A integral definida na Eq. (3) é uma integral tabelada [6] que, resolvida e após uma pequena manipulação algébrica, nos dá

$$
S=\frac{v_{0}^{2}}{g}\left[\operatorname{sen} \theta+\cos ^{2} \theta \ln \left(\frac{1+\operatorname{sen} \theta}{\cos \theta}\right)\right] .
$$

A Eq. (4) é a mesma equação obtida, na referência [2], quando $d S$ foi integrada em $x$.

\section{b) Modelo com resistência do ar}

Assumindo-se uma força de resistência com o ar da forma $\vec{F}=-b \vec{v}$, as equaçốes do movimento do projétil são escritas como

$$
\ddot{x}=-k \dot{x}
$$

$$
\ddot{y}=-k \dot{y}-g .
$$

Onde $k=b / m, m$ é a massa do projétil e $g$ é a aceleração da gravidade. As equações (5a) e (5b) podem ser integradas facilmente $[7,8]$ fornecendo

$$
\dot{x}=v_{0} \cos \theta e^{-k t},
$$

e

$$
\dot{y}=\left(v_{0} \operatorname{sen} \theta+\frac{g}{k}\right) e^{-k t},
$$

Integrando as equações (6a) e (6b), obtemos as posições $x$ e $y$ :

$$
x=\frac{v_{0} \cos \theta}{k}\left(1-e^{-k t}\right),
$$

$$
y=\frac{1}{k}\left(v_{0} \operatorname{sen} \theta+\frac{g}{k}\right)\left(1-e^{-k t}\right)-\frac{g}{k} t .
$$

A equação da trajetória pode ser encontrada eliminando-se o tempo entre as equações (7a) e (7b), obtendo-se

$$
y=\left(\operatorname{tg} \theta+\frac{g}{k v_{0} \cos \theta}\right) x+\frac{g}{k^{2}} \ln \left(1-\frac{k x}{v_{0} \cos \theta}\right)
$$

O tempo de subida do projétil é determinado da Eq. (6b) $\operatorname{com} \dot{y}=0$,

$$
t_{s}=\frac{1}{k} \ln \left(1+\frac{k v_{0} \operatorname{sen} \theta}{g}\right)
$$

Substituindo o valor de $t_{s}$ na Eq. (7b), determinamos a altura máxima atingida pelo projétil

$$
H=\frac{v_{0} \operatorname{sen} \theta}{k}-\frac{g}{k^{2}} \ln \left(1+\frac{k v_{0} \operatorname{sen} \theta}{g}\right) .
$$

Para estabelecermos o comprimento da trajetória procederemos como no caso do modelo parabólico, integraremos no tempo. Usando as equações (6a) e (6b) escrevemos

$$
\frac{d y}{d x}=\frac{(k u+g)-g e^{-k t}}{k v}
$$

onde $u$ e $v$ são definidas como anteriormente. Substituindo a Eq. (11) na Eq. (1) e integrando no tempo temos, 


$$
S=\int_{0}^{T} \frac{\sqrt{k^{2} v^{2}+(k u+g)^{2}-2 g(k u+g) e^{k t}+g^{2} e^{2 k t}}}{k v}\left(\frac{v}{e^{k t}}\right) d t
$$

Fazendo-se uma mudança de variável, $\chi=e^{k t}$ e definindo $c=k^{2} v^{2}+(k u+g)^{2}, b=-2 g(k u+g)$ e $a=g^{2}$, a Eq. (12) pode ser escrita como

$$
S=\frac{1}{k^{2}} \int_{1}^{\varepsilon} \frac{\sqrt{a \chi^{2}+b \chi+c}}{\chi^{2}} d \chi
$$

onde $\varepsilon=e^{k T}$ e $T$ é o tempo de vôo. A integral definida na Eq. (13) é uma integral tabelada [6] que, resolvida, nos fornece

$$
\begin{gathered}
S=\frac{1}{k^{2}}\left\{\sqrt{a+b+c}-\sqrt{\frac{a \varepsilon^{2}+b \varepsilon+c}{\varepsilon}}+\sqrt{a} \ln \left(\frac{2 \sqrt{a} \sqrt{a \varepsilon^{2}+b \varepsilon+c}+2 a \varepsilon+b}{2 \sqrt{a} \sqrt{a+b+c}+2 a+b}\right)\right. \\
\left.+\frac{b}{2 \sqrt{c}} \ln \left[\frac{(2 \sqrt{c} \sqrt{a+b+c}+b+2 c) \varepsilon}{2 \sqrt{c} \sqrt{a \varepsilon^{2}+b \varepsilon+c}+b \varepsilon+2 c}\right]\right\} .
\end{gathered}
$$

Para obtermos valores de $S$, é necessário conhecermos o tempo de vôo $T$. O valor de $T$ pode ser calculado através da Eq. (7b) admitindo-se $y=0$. Ou seja, o tempo de vôo satisfaz a equação transcendental

$$
g T=\left(u+\frac{g}{k}\right)\left(1-e^{-k T}\right),
$$

que pode ser resolvida graficamente ou usando-se um método numérico tal como o método de Newton [9]. $\mathrm{Na}$ seção seguinte, faremos comparações entre os resultados do modelo parabólico, Eq. (4) e o modelo com força de resistência do ar, Eq. (14).

\section{Resultados}

Para verificarmos a exatidão da Eq. (14), integramos numericamente a Eq. (13) usando uma rotina baseada no algoritmo de Romberg [10]. Fizemos também um cálculo aproximado de $S$ usando, na Eq. (14), o tempo de vôo obtido de uma lei empírica, formulada por Littlewood [11], que tem a seguinte forma:

$$
T_{L}=\sqrt{\frac{8 H}{g}} .
$$

$H$ é a altura máxima atingida pelo projétil e $g$ é a aceleração da gravidade. J. E. Littlewood foi um pesquisador inglês que estudou exaustivamente os problemas de balística durante a primeira grande guerra mundial e estabeleceu a lei empírica definida na Eq. (16) para o movimento de projéteis lançados da superfície da terra. É fácil verificar que a Eq. (16) é exata quando o movimento do projétil é parabólico. Podemos investigar a natureza da fórmula (16) no presente caso no regime de $k T<<1$. Inicialmente, expandimos a exponencial na Eq. (15) para determinarmos o tempo de vôo em sucessivas aproximações [7,8], obtendo

$$
T=\frac{k u+g}{g k}\left(k T-\frac{k^{2} T^{2}}{2}+\frac{k^{3} T^{3}}{6}-\cdots\right)
$$

Dividindo por $T$, podemos escrever a Eq. (16) como,

$$
T=\frac{2 u}{k u+g}+\frac{k T^{2}}{3}
$$

No limite $k \rightarrow 0$ (modelo parabólico), a Eq. (17) nos fornece o tempo de vôo correspondente, $T_{0}=2 u / g$. De modo que, se $k$ é bem pequeno mas não zero, o tempo de vôo seria aproximadamente igual a $T_{0}$. Se usarmos este valor aproximado na Eq. (17), obteremos o termo seguinte em primeira ordem em $k$ :

$$
T_{1}=\frac{2 u}{g}\left[\left(1+\frac{k u}{g}\right)^{-1}+\frac{2 k u}{3 g}\right]
$$

Para avaliarmos o termo $k u / g$ em comparação a $k T$, calculamos $k u / g$ e $k T$ para diferentes valores de $v_{0}$ e $k$, em função do ângulo de lançamento. Na Fig. 2, mostramos um resumo desses cálculos. 


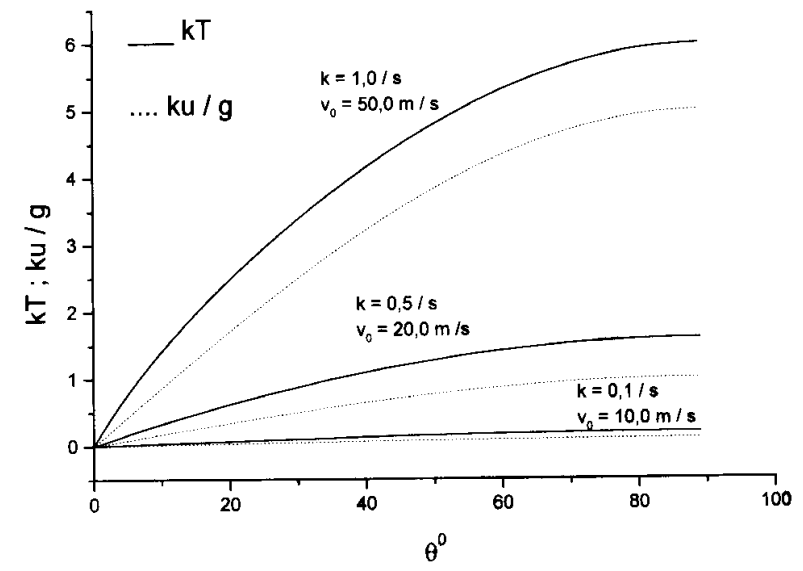

Figura 2. Curvas de $k u / g$ e $k T$ em função do ângulo de lançamento.

Podemos verificar que, para todos os casos, $k u / g<k T$ de modo que podemos expandir o termo entre parênteses, Eq. 18, e escrever

$$
T_{1}=\frac{2 u}{g}-\frac{2 k u^{2}}{3 g^{2}}
$$

A Eq. (20) nos dá o tempo de vôo em primeira ordem. Mostraremos agora que podemos determinar a Eq. (20) a partir da lei de Littlewood. Usando o valor de $H$, Eq. (10), na Eq. (16) e expandindo o logaritmo, temos

$$
T_{L 1}=\sqrt{\frac{8}{g}\left[\frac{u}{k}-\frac{g}{k^{2}}\left(\frac{k u}{g}-\frac{k^{2} u^{2}}{2 g^{2}}+\frac{k^{3} u^{3}}{3 g^{3}}-\cdots\right)\right]} .
$$

Considerando termos até primeira ordem, ficamos com

$$
T_{L 1}=\frac{2 u}{g} \sqrt{1-\frac{2 k u}{3 g}}
$$

que, expandindo a raiz quadrada, nos fornece

$$
T_{L 1}=\frac{2 u}{g}\left(1-\frac{1}{2} \frac{2 k u}{3 g}\right)=\frac{2 u}{g}-\frac{2 k u^{2}}{3 g^{2}}
$$

que é o mesmo resultado da Eq. (20). Vemos, então, que a lei empírica de Littlewood é, para o caso de resistência do ar proporcional à velocidade do projétil, uma expansão em potências de $k$ e que coincide em primeira ordem com o valor exato para $k$ pequeno.

Numericamente, podemos estudar a discrepância entre a lei de Littlewood e os resultados exatos do presente modelo, definindo o erro percentual relativo como:

$$
\Delta=\left(\frac{T-T_{L}}{T}\right) \times 100 \%
$$

Na tabela 1 , mostramos valores de $\Delta$ para diferentes valores de $k$, de $v_{0}$ e do ângulo de lançamento. Podemos verificar que, para $k<1,0$, os erros percentuais

\begin{tabular}{|c|c|c|c|c|c|c|}
\hline \multirow[t]{3}{*}{$\theta^{0}$} & \multicolumn{6}{|c|}{$\Delta \%$} \\
\hline & \multicolumn{3}{|c|}{$v=10,0 \mathrm{~m} / \mathrm{s}$} & \multicolumn{3}{|c|}{$k=0,1 / s$} \\
\hline & $k=0,1 / s$ & $k=0.5 / \mathrm{s}$ & $k=1,0 / s$ & $v=5,0 \mathrm{~m} / \mathrm{s}$ & $v=20,0 \mathrm{~m} / \mathrm{s}$ & $v=50,0 \mathrm{~m} / \mathrm{s}$ \\
\hline 5 & 0,0002 & 0,0051 & 0,0199 & 0,0001 & 0,0008 & 0,0051 \\
\hline 25 & 0,0048 & 0,1088 & 0,3885 & 0,0012 & 0,0188 & 01088 \\
\hline 45 & 0,0133 & 0,2818 & 0,9508 & 0,0034 & 0,05033 & 0,2818 \\
\hline 65 & 0,0215 & 0,4398 & 1,4358 & 0,0055 & 0,0215 & 0,4398 \\
\hline 85 & 0,0259 & 0,5198 & 1,6737 & 0,0067 & 0,0259 & 0,5197 \\
\hline
\end{tabular}
relativos são menores que $1,0 \%$, indicando que neste regime a fórmula de Littlewood nos fornece tempos de vôo com precisão desta ordem.

Tabela 1. Valores do erro relativo, (eq. 20)

Na tabela 2, mostramos uma comparação entre o resultado aproximado, usando a lei de Littlewood para o tempo de vôo na Eq. (14), e o resultado da integração numérica e analítica com o tempo de vôo obtido da Eq. (15) resolvida pelo método de Newton. Adotando o sistema MKS, os valores $k=0,1 ; 0,5 ; 1,0 / s, v_{0}=20,0$ $\mathrm{m} / \mathrm{s}$ e $g=10,0 \mathrm{~m} / \mathrm{s}^{2}$ são assumidos para diversos valores do ângulo de lançamento. Observando a tabela 2 , notamos que os resultados analíticos e numéricos são iguais no número de figuras apresentadas, e o resultado aproximado apresenta uma discrepância, crescente com o valor de $k$, em relação ao cálculo numérico e analítico. Como era de se esperar para $k=0,1 / s$, o erro relativo máximo é de apenas de 0,05\%; no caso de $k=0,5 / s$; o erro relativo máximo é de $0,89 \%$; e para $k=1,0 / \mathrm{s}$, o erro relativo máximo sobe para $2,5 \%$.

Na Fig. 3, mostramos o gráfico do comprimento da trajetória $(S)$ e do alcance $(R)$ em função do ângulo de lançamento para o modelo parabólico $(k=0)$ e o modelo com resistência do ar $(k=0,5 / s)$. Assumimos 
$v_{0}=10,0 \mathrm{~m} / \mathrm{s}$ e $g=10,0 \mathrm{~m} / \mathrm{s}^{2}$. Claramente, o gráfico mostra o efeito da resistência do ar no comprimento da trajetória e no alcance. Para ângulos de lançamentos maiores do que $10^{\circ}$, a diferença entre os comprimentos das trajetórias e dos alcances acentua-se e as trajetórias máximas correspondentes diferem em mais de $30 \%$. Os alcances máximos, por sua vez, diferem em mais de 32 $\%$.

Para lançamentos verticais $(\theta=\pi / 2)$ os comprimentos das trajetórias podem ser obtidos facilmente e podemos determinar a diferença de comprimento entre as duas trajetórias pela seguinte expressão:

$$
\Delta S=S_{0}(\pi / 2)-S_{k}(\pi / 2)=\frac{2 g}{k^{2}} \ln \left(1+\frac{k v_{0}}{g}\right)+\frac{v_{0}^{2}}{g}-\frac{2 v_{0}}{k} .
$$

Tabela 2. Comparação do comprimento da trajetória de um projétil. Cálculo aproximado, numérico e analítico. $\nu_{0}=10,0 \mathrm{~m} / \mathrm{s}, g=10,0 \mathrm{~m} / \mathrm{s}^{2}$.

\begin{tabular}{|c|c|c|c|}
\hline \multicolumn{4}{|c|}{$S(m)$} \\
\hline$\theta^{o}$ & Aproximado & $\begin{array}{l}\text { Numérico } \\
1 / s\end{array}$ & Analitico \\
\hline 5 & 1,719 & 1,719 & 1,719 \\
\hline 15 & 4,892 & 4,892 & 4,892 \\
\hline 25 & 7,518 & 7,518 & 7,518 \\
\hline 35 & 9,439 & 9,440 & 9,440 \\
\hline 45 & 10,592 & 10,594 & 10,594 \\
\hline 55 & 11,007 & 11,010 & 11,010 \\
\hline 65 & 10,806 & 10,810 & 10,810 \\
\hline 75 & 10,203 & 10,208 & 10,208 \\
\hline 85 & 9,538 & 9,543 & 9,543 \\
\hline \multicolumn{4}{|c|}{$k=0,5 / s$} \\
\hline 5 & 1,643 & 1,643 & 1,643 \\
\hline 15 & 4,309 & 4,310 & 4,310 \\
\hline 25 & 6.195 & 6,201 & 6,201 \\
\hline 35 & 7,407 & 7,421 & 7,421 \\
\hline 45 & 8,070 & 8,094 & 8,094 \\
\hline 55 & 8,299 & 8,337 & 8,337 \\
\hline 65 & 8,206 & 8,257 & 8,257 \\
\hline 75 & 7,911 & 7,972 & 7,972 \\
\hline 85 & 7,578 & 7,646 & 7,646 \\
\hline \multicolumn{4}{|c|}{$k=1,0 / s$} \\
\hline 5 & 1,555 & 1,556 & 1,556 \\
\hline 15 & 3,734 & 3,738 & 3,738 \\
\hline 25 & 5,042 & 5,058 & 5,058 \\
\hline 35 & 5,800 & 5,834 & 5,834 \\
\hline 45 & 6,202 & 6,261 & 6,261 \\
\hline 55 & 6,357 & 6,446 & 6,446 \\
\hline 65 & 6,334 & 6,453 & 6,452 \\
\hline 75 & 6,195 & 6,338 & 6,338 \\
\hline 85 & 0,024 & 6,180 & 6,180 \\
\hline
\end{tabular}

Podemos notar que, no limite $k \rightarrow 0$, temos que $\Delta S \rightarrow 0$ e quando $k \rightarrow \infty, \Delta S \rightarrow \frac{v_{0}^{2}}{g}$, que é o valor do comprimento da trajetória do modelo parabólico. Para o caso mostrado na Fig. 3, a Eq. (22) nos dá $\Delta S=$ $2,44 \mathrm{~m}$, que está de acordo com o valor calculado na figura.

Nas Figs. 4 e 5, mostramos os gráficos de um estudo do comprimento da trajetória e do alcance em função do parâmetro $k$. Apresentamos curvas de $S$ e $R$ em função de $\theta$ para $k=0,1 ; 0,5 ; 1,0 / \mathrm{s}$ e $v_{0}=10,0 \mathrm{~m} / \mathrm{s}$. Nas 
Figs. 5 e 7 temos agora os resultados de $S$ e $R$ para $k$ $=0,5 / \mathrm{s}$ e diferentes velocidades de lançamento: $v_{0}=$ 5,$0 ; 10,0$ e $20,0 \mathrm{~m} / \mathrm{s}$.

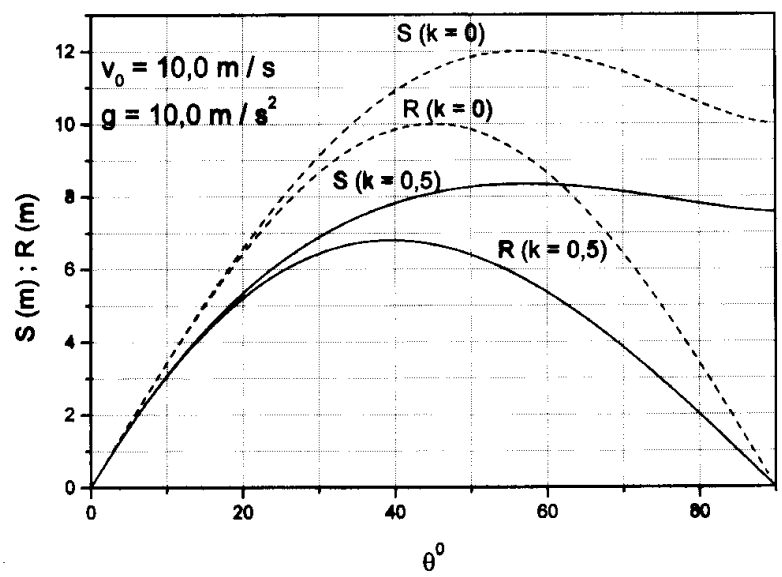

Figura 3. Gráfico de $\mathrm{S}$ e $\mathrm{R}$ em função do ângulo de lançamento para o modelo parabólico (linha pontilhada) e para o modelo com resistência do ar (linha contínua).

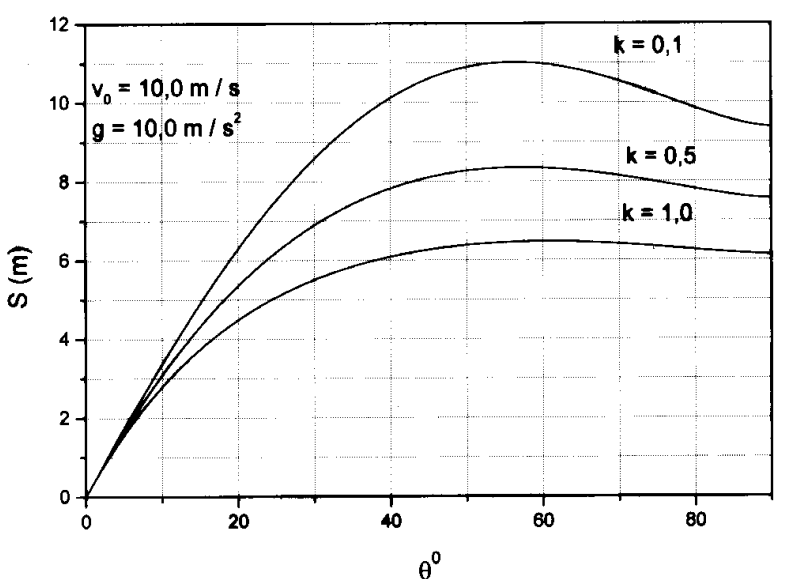

Figura 4. Curvas de S em função do ângulo de lançamento para diferentes valores de $k$.

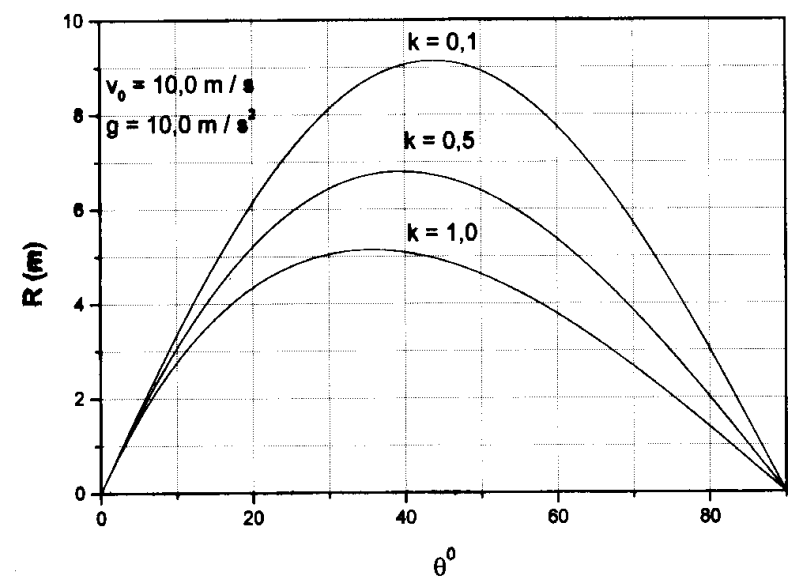

Figura 5. Curvas de $R$ em função do ângulo de lançamento para diferentes valores de $k$.

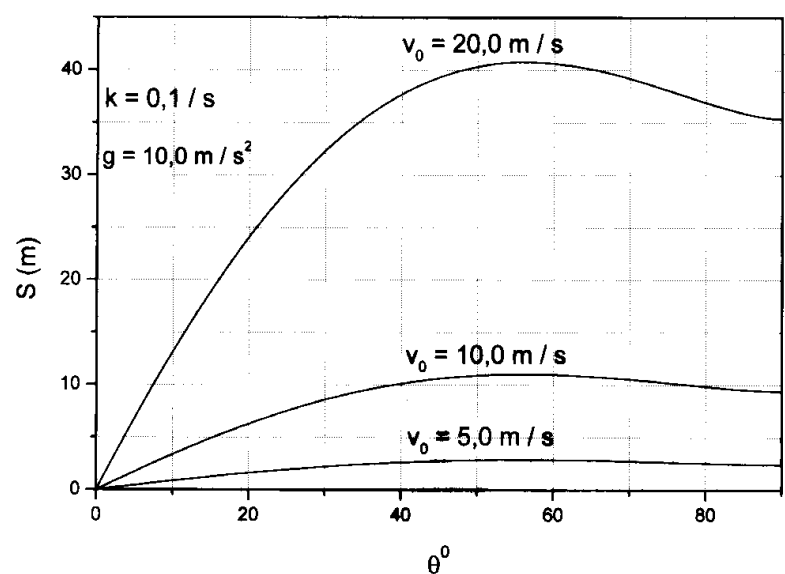

Figura 6. Curvas de $S$ em função do ângulo de lançamento para diferentes velocidades iniciais. $k=0,1 / \mathrm{s}$.

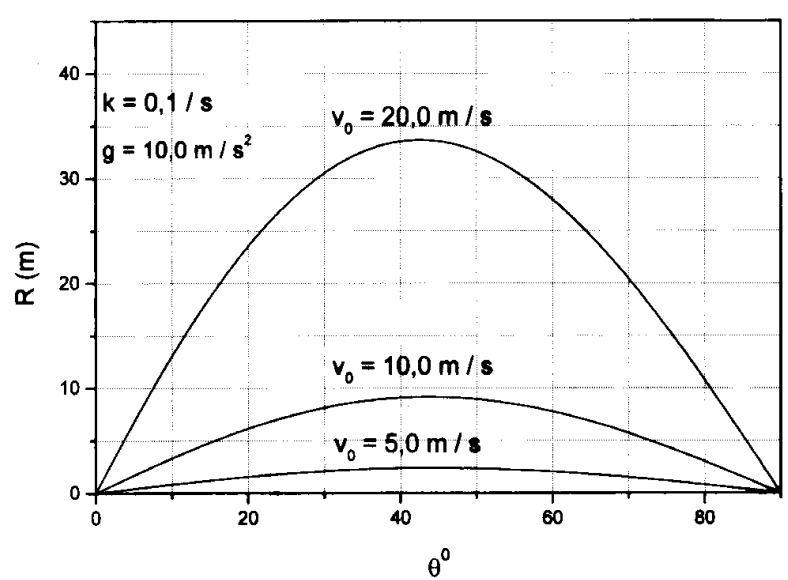

Figura 7. Curvas de $R$ em função do ângulo de lançamento para diferentes velocidades iniciais. $k=0.1 / \mathrm{s}$.

Fazendo $d S / d \theta=0$ na Eq. (4), podemos determinar o ângulo de lançamento, que produz a trajetória de maior comprimento no modelo parabólico, para uma dada velocidade de lançamento, ou seja,

$$
\operatorname{sen} \theta_{\text {max }} \ln \left(\frac{1+\operatorname{sen} \theta_{\text {max }}}{\cos \theta_{\text {max }}}\right)=1
$$

A Eq. (23) é uma equação transcendental cuja solução pode ser obtida graficamente ou, por exemplo, pelo método de Newton [7]. Resolvendo (23) pelo método de Newton, obtivemos, para o modelo parabólico, o ângulo de lançamento que produz a trajetória de maior comprimento independentemente do valor da velocidade de lançamento. $\mathrm{O}$ valor de $\theta_{\max }$ calculado com dezesseis figuras é

$\theta_{\max }=56,465835127452350^{\circ}$.

Para obtermos $\theta_{\text {max }}$ para o modelo com atrito derivamos numericamente a curva $S(\theta)$ e verificamos o ângulo para o qual a derivada se anula. Na Fig. 8, temos a ilustração desse procedimento para o caso de $k$ $=0,1 / \mathrm{s}$. 


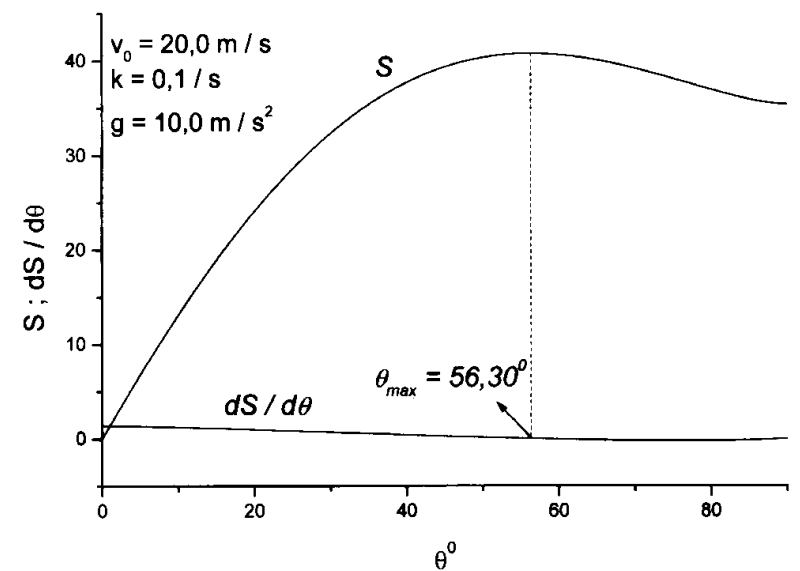

Figura 8. Gráfico de $\mathrm{S}$ e $d S / d \theta$ em função do ângulo de lançamento.

Finalmente, na Fig. 9, apresentamos os valores máximos do comprimento da trajetória, do alcance em função de $k$ e mostramos também os valores correspondentes de $\theta_{\max }$. A velocidade de lançamento foi: $v_{0}=$ $20,0 \mathrm{~m} / \mathrm{s}$. É interessante notar que, quando $k$ cresce, o ângulo de lançamento que dá $R$ máximo diminui; enquanto que, para o comprimento da trajetória, o ângulo que dá $S$ máximo aumenta para $v_{0}$ fixa.

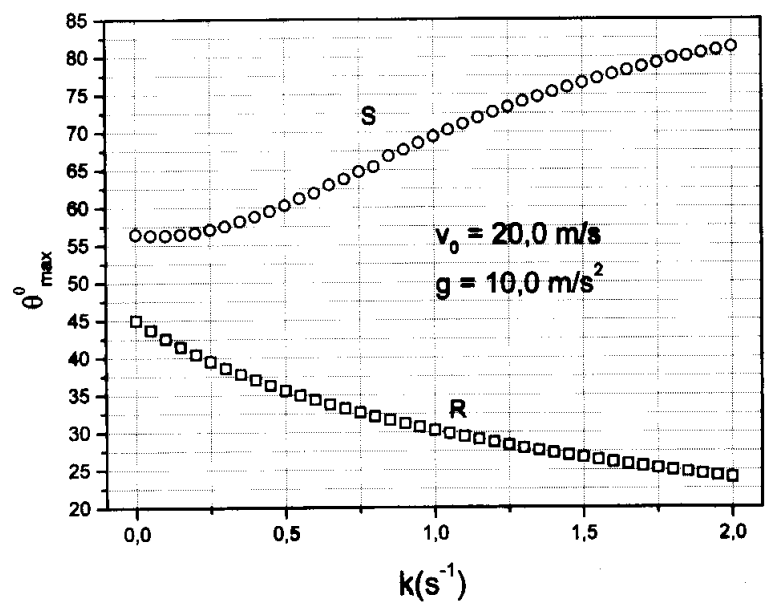

Figura 9. Valores do comprimento máximo da trajetória, do alcance máximo, $\theta_{\max }$ e $k$.

\section{Conclusões}

A determinação do movimento de um projétil lançado da superfície da terra é um problema importante na história da mecânica. O modelo mais simples, movimento do projétil na ausência da atmosfera, é abordado nos cursos introdutórios de física como um exemplo importante de movimento em duas dimensões. A presença da atmosfera introduz forças de atrito que complicam a obtenção das soluções para este problema. Neste trabalho analisamos um modelo de movimento de projétil que, além da força gravitacional, inclui uma força de arraste diretamente proporcional à velocidade do projétil. Resolvemos analiticamente as equações do movimento e derivamos uma expressão para o comprimento da trajetória. No desenvolvimento deste trabalho, tanto o tratamento analítico como o uso de métodos numéricos foram necessários e podem ser implementados como exemplo, nos cursos básicos de mecânica, ensejando ao estudante experiência teórica e computacional.

\section{Agradecimentos}

Agradecemos ao Prof. Lindberg L. Gonçalves pelas valiosas discussões e ao árbitro pelas sugestões. Este trabalho foi financiado parcialmente pela FINEP.

\section{Referências}

[1] S. Drake, J. MacLachlan, Scentific American, março, (1975).

[2] H. Serafian, The Phys. Teacher, fevereiro, (1999).

[3] J. Fazuri, J. M. O. Matos, Rev. Bras. de Ensino de Física, 20, 327 (1998).

[4] S. Timoshenko, D. H. Young, Advanced Dynamics, McGraw-Hill Book Company, Inc. (1948).

[5] Timmerman, Jacobus P. van der Weele, Am. J. Physics, 67, 538 (1999)

[6] M. S. Spiegel, Manual de fórmulas, métodos e tabelas de matemática, Makron, McGraw-Hill, (Coleção Schaum),Segunda edição, pg. 107 (1992).

[7] K. R. Symon, Mechanics, Third Edition, AddisonWesley, (1971).

[8] J. B. Marion, S. T. Thorton, Classical Dynamics of Particles and Systems, Saunders College Publishing, (1970).

[9] P. L. DeVries, A first Course in Computational Physics, Wiley, (1994).

[10] W. H. Press, S. A. Teukolsky, B. P. Flannery, W. T. Vetterling, Numerical Recipes, the art of scientific computing, Cambridge University Press, Second Edition, (1992).

[11] J. E. Littlewood, A Mathematician's Miscellany, Mathuen, London (1953); Math. Gazette, 52, 132 (1968); Adventures in ballistics I, II (1915-1918): Math. Spectrum, 4, 31 (1971). 